\title{
AIP
}

\section{Transition state spectroscopy on the Li-HF system}

Miguel Paniagua, Alfredo Aguado, Manuel Lara, and Octavio Roncero

Citation: J. Chem. Phys. 109, 2971 (1998); doi: 10.1063/1.476886

View online: http://dx.doi.org/10.1063/1.476886

View Table of Contents: http://jcp.aip.org/resource/1/JCPSA6/v109/i8

Published by the American Institute of Physics.

\section{Additional information on J. Chem. Phys.}

Journal Homepage: http://jcp.aip.org/

Journal Information: http://jcp.aip.org/about/about_the_journal

Top downloads: http://jcp.aip.org/features/most_downloaded

Information for Authors: http://jcp.aip.org/authors

\section{ADVERTISEMENT}

\section{Instruments for advanced science}
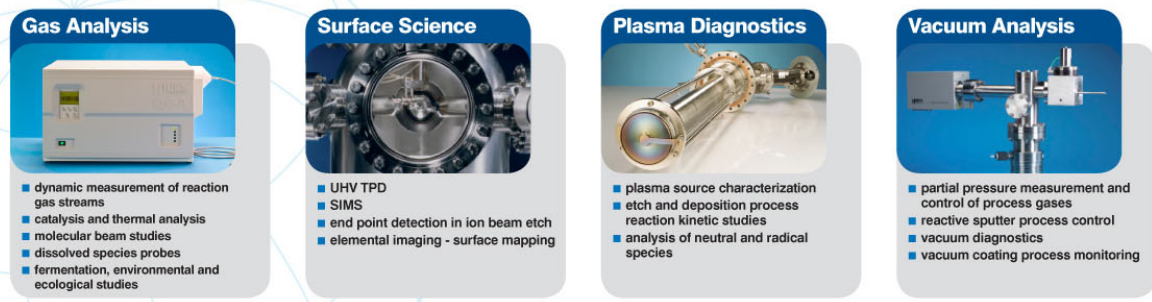

contact Hiden Analytical for further details

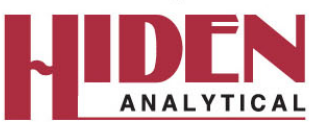

info@hideninc.com www.HidenAnalytical.com CLICK to view our product catalogue 


\title{
Transition state spectroscopy on the Li-HF system
}

\author{
Miguel Paniagua \\ Departamento de Química Física, Facultad de Ciencias C-XIV, Universidad Autónoma de Madrid, \\ 28049 Madrid, Spain
}

Alfredo Aguado, a) Manuel Lara, and Octavio Roncero

Instituto de Matemáticas y Física Fundamental, C.S.I.C., Serrano 123, 28006 Madrid, Spain

(Received 16 March 1998; accepted 19 June 1998)

\begin{abstract}
The transition state region of the Li-HF system is theoretically studied via infrared excitation of the ground state of the complex in the reactant valley. The absorption spectrum shows intense peaks for which $\mathrm{LiF}$ is produced with high efficiency $(\approx 90 \%)$, while the reaction has a very low cross section during the collision at the same energies. The reason is that the resonances reached through optical excitation are in the vicinity of the transition state. (C) 1998 American Institute of Physics.
\end{abstract}

[S0021-9606(98)01432-9]

Transition state spectroscopy (TSS) of bimolecular reactions provides direct information on the short lived resonances at precise energies at the region where chemical change occurs and, therefore, on the mechanisms governing these processes. Negative ion photoelectron spectroscopy, ${ }^{1,2}$ and spectroscopy of weakly bonded complexes of the reactants $^{3-10}$ are two kinds of experimental TSS studies. Much attention has been paid to the TSS experimental studies on M-HX systems $(\mathrm{M}=$ alkali or alkaline earth atom, $\mathrm{X}=$ halogen atom) because they usually present a van der Waals well in the reactants valley, from which the system is promoted to an electronically excited state of the metal atom, as it is the case of $\mathrm{Ca}-\mathrm{HCl}$ and $\mathrm{Ca}-\mathrm{HBr}{ }^{5,6}$ The excited complex evolves towards the products channel in the presence of several electronic states, which are often connected by nonadiabatic couplings. Moreover, the theoretical modeling of such dynamics is, nowadays, very difficult since it involves the calculation, within spectroscopic accuracy, of several excited electronic states, with their mutual nonadiabatic couplings.

In the ground electronic state, however, most of the studies on the reactivity of these systems correspond to the $\mathrm{HX}+\mathrm{M}$ collision. Due to the contribution of many partial waves to the collisional total reaction cross section, the experimental detection of resonances is complicated, and these are the structures associated to the transition state of significant interest. However, these systems are good candidates for TSS studies on the ground electronic state via infrared absorption from a bound complex for several reasons. First, most of these systems present a deep well in the reactant channel, which makes possible the formation of a complex between reactants. Second, these reactions are envisaged as the transfer of an electron of the alkali atom to the halogen atom $^{11}$ ("harpoon" mechanism) at precise configurations. Such rapid electronic transfer produces a sudden change in the dipolar moment on the ground electronic state and makes

\footnotetext{
${ }^{a)}$ Permanent address: Departamento de Química Física, Facultad de Ciencias C-XIV, Universidad Autónoma de Madrid, 28049 Madrid, Spain.
}

possible the vibrational excitation. These two conditions are commonly valid for a wide variety of systems where the "harpoon" mechanism is applicable, and therefore, the infrared excitation of the dimer complex can be used to analyze the transition region.

Moreover, these systems show a very important enhancement of the reactivity with the initial vibrational excitation of the $\mathrm{HX}$ reactant, from $v=0$ to 1 , as it is the case for $\mathrm{K}-\mathrm{HCl},{ }^{12} \mathrm{Ba}-\mathrm{HF},{ }^{13} \mathrm{Ca}-\mathrm{HF},{ }^{14}$ and $\mathrm{K}-\mathrm{HF} .{ }^{15}$ These reactions are mostly endothermic or nearly thermoneutral and present a late barrier, that explains the effect of the initial vibration of the reactants on the efficiency of the reaction. ${ }^{16,17}$ Thus, an excitation of the HX vibration during the infrared transition of the complex can lead the system close to the transition state region.

In this work we envisage the study of Li-HF as a prototype of these systems since it is the lightest one and a high quality potential energy surface (PES) has recently been published $^{18,19}$ which yields reaction cross sections in good agreement with the available experimental data on the collision process. ${ }^{20}$ This PES is based on accurate $a b$ initio points ${ }^{21}$ and has two wells, in the reactant and products channels, respectively. The ground bound state of the complex is located in the entrance channel at $\approx 2109 \mathrm{~cm}^{-1}$ below the $\operatorname{HF}(v=0, j=0)$ asymptote and a contour plot of it is presented in Fig. 1(a).

The photoinitiated reaction dynamics is studied in the framework of the first order perturbation theory for electric dipole transitions, the transition operator being $\mathbf{d} \cdot \mathbf{e}$, where $\mathbf{e}$ is the polarization vector of the incident light while $\mathbf{d}$ is the electric dipole moment on the ground electronic state. The initial wave packet is formed by applying $\mathbf{d} \cdot \mathbf{e}$ to the ground eigenstate of the complex, of $J_{i}$ total angular momentum, and projecting on the final $J$, to consider a $J_{i} \rightarrow J$ transition. The wave packet is represented on reactant Jacobi coordinates as described in Ref. 19, using a body-fixed frame in which the $z$-axis lies along the $\mathbf{R}$ vector, the distance between the Li atom and HF center of mass, and the HF internuclear distance $\mathbf{r}$ is in the $x-z$ plane.

The $\mathbf{d}$ body-fixed Cartesian components are calculated at 


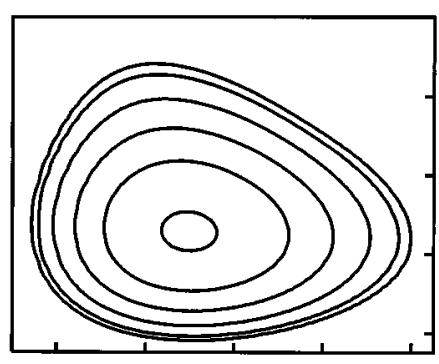

1.5

1.1

0.9
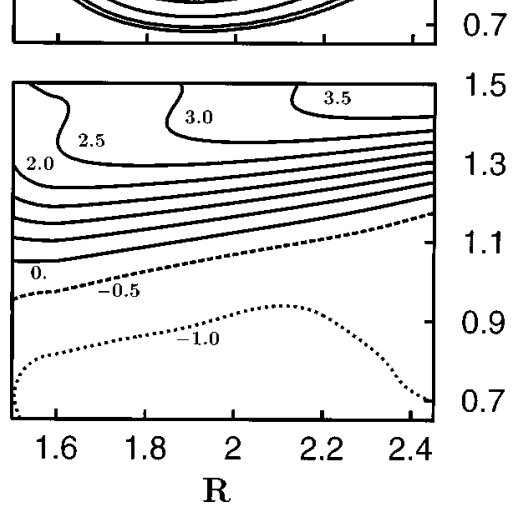

1.5

$1.1 \mathrm{r}$

FIG. 1. (a) Contour plot of the probability density of the ground state of the complex with $J_{i}=1$ (each contour is a tenth of the previous one); (b) $\mathbf{d}_{z}(r, R, \gamma)$ (in atomic units) component for $\gamma=106^{\circ}$. Distances are in angstroms.

the same level of accuracy as the $a b$ initio points ${ }^{21}$ used to fit the PES. ${ }^{19}$ As expected, the two components in the bodyfixed plane present a sharp change due to the crossing between covalent and ionic states, responsible for the electron transfer. This fact complicates the fit of these components to an analytic function over the entire space defined by the internal coordinates, $r, R$, and $\gamma$. For this reason, the fit has been performed only in the region where the ground state of the complex is localized and the result is shown in Fig. 1(b) for the main component $\mathbf{d}_{z}$.

The largest slope in the electric dipole moment is along the HF internuclear distance. The initial wave packet thus obtained presents a significant excitation on the HF vibration, Fig. 2(a), and it is located in the neighborhood of the transition state, in Fig. 2(b). The main component of the wave packet corresponds to the ground state, with a probability of $\approx 72 \%$, that remains bound during the time evolution. In order to avoid numerical difficulties during the propagation, the first 7 bound levels of the complex are subtracted from the initial wave packet, to perform the time integration. Those bound states are calculated in a variational way using a large basis set (of 4000 functions).

The total absorption spectrum, $\sigma(E)$, is obtained as the Fourier transform of the autocorrelation function, and is presented in Fig. 3(a). It consists of an intense band with two major peaks about $0.08-0.18 \mathrm{eV}$ above the $\operatorname{HF}(v=0, j$ $=0$ ) asymptote. Since the ground bound state energy of the complex is $\approx-0.26 \mathrm{eV}$, this band corresponds to a transition of $\approx 0.34-0.44 \mathrm{eV}$, which is lower than that of free HF, $\approx 0.5 \mathrm{eV}$. Therefore, this band is mainly associated with an excitation of one vibrational quantum in the HF vibration mode. In addition, a second band was found at $0.4-0.65 \mathrm{eV}$, i.e., $\approx 0.4 \mathrm{eV}$ above the first band, of much lower intensity and that corresponds to a $v=2$ excitation of the HF vibra-

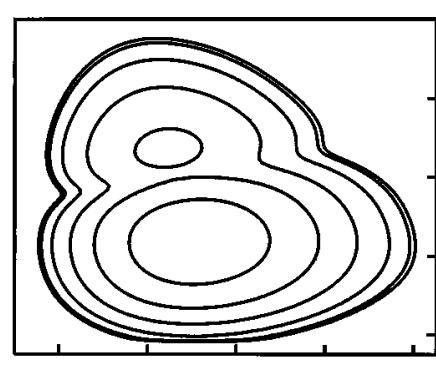

1.5

1.3

$1.1 \mathrm{r}$

0.9

0.7

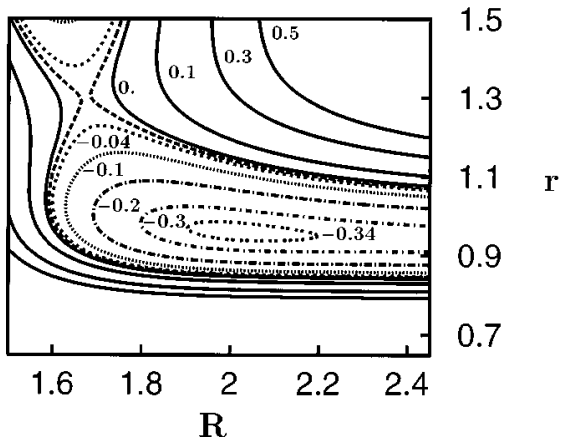

FIG. 2. (a) Contour plot of the probability density of the initial wave packet for the $J_{i}=1 \rightarrow J=0$ transition (each contour is a tenth of the previous one), and (b) contour plot of the PES (in eV referred to $\operatorname{HF}(v=0, j=0)$ ) at $\gamma$ $=73^{\circ}$. Distances are in angstroms.

tional mode. The reduction of the frequency of this mode within the complex, as compared to the one of free HF, can be explained by looking at Fig. 2(b), where the width of the well for the HF vibration increases as HF gets closer to the $\mathrm{Li}$ atom. The other two internal vibrations are also excited since the dipole moment components show significant changes along $R$ and $\gamma$. An interesting point is that this band, shown in Fig. 3(a), is close to the reaction threshold approximately at $0.068 \mathrm{eV}$ (which corresponds to the saddle point energy, $-0.021 \mathrm{eV}$, plus the zero point energy ${ }^{19}$ ).

The partial cross sections, $\sigma_{v j}^{\mathrm{HF}}(E)$, on each of the $\operatorname{HF}(v$, $j$ ) asymptotic states are calculated by the method proposed by Balint-Kurti et al., ${ }^{22}$ using reactant Jacobi coordinates, while the partial cross sections on the $\operatorname{LiF}\left(v^{\prime}, j^{\prime}\right)$ asymptotic states are obtained using product Jacobi coordinates. The absorption spectrum obtained in the two cases are in very good agreement. In order to converge the partial cross sections, the propagations in the two sets of Jacobi coordinates were performed until $3 \mathrm{ps}$, so that $\approx 98.3 \%$ of the wave packet is absorbed. The wave packet is not completely absorbed because small components on some bound states, above the first seven already subtracted, remain in the initial wave packet. In addition, the sum over all the partial cross sections, in reactants and products, is equal, within $1 \%$, to the total absorption spectrum.

The reaction cross section obtained as the sum over all final $\operatorname{LiF}\left(v^{\prime}, j^{\prime}\right)$ states, in Fig. 3(a), accounts for $\approx 88 \%-92 \%$ of the total absorption probability and $\mathrm{LiF}$ products are mainly populated in the $v^{\prime}=0$ and 1 states, the $v^{\prime}=2$ population being nearly negligible. The LiF population in $v^{\prime}=0$, although being the dominant one at $E$ $=0.08 \mathrm{eV}$, with a normalized weight of $92 \%$, decreases monotonically with energy, to nearly $65 \%$ for $E=0.18 \mathrm{eV}$. The rotational distribution of $\operatorname{LiF}\left(v^{\prime}=0\right)$, in Fig. 3(b), is 

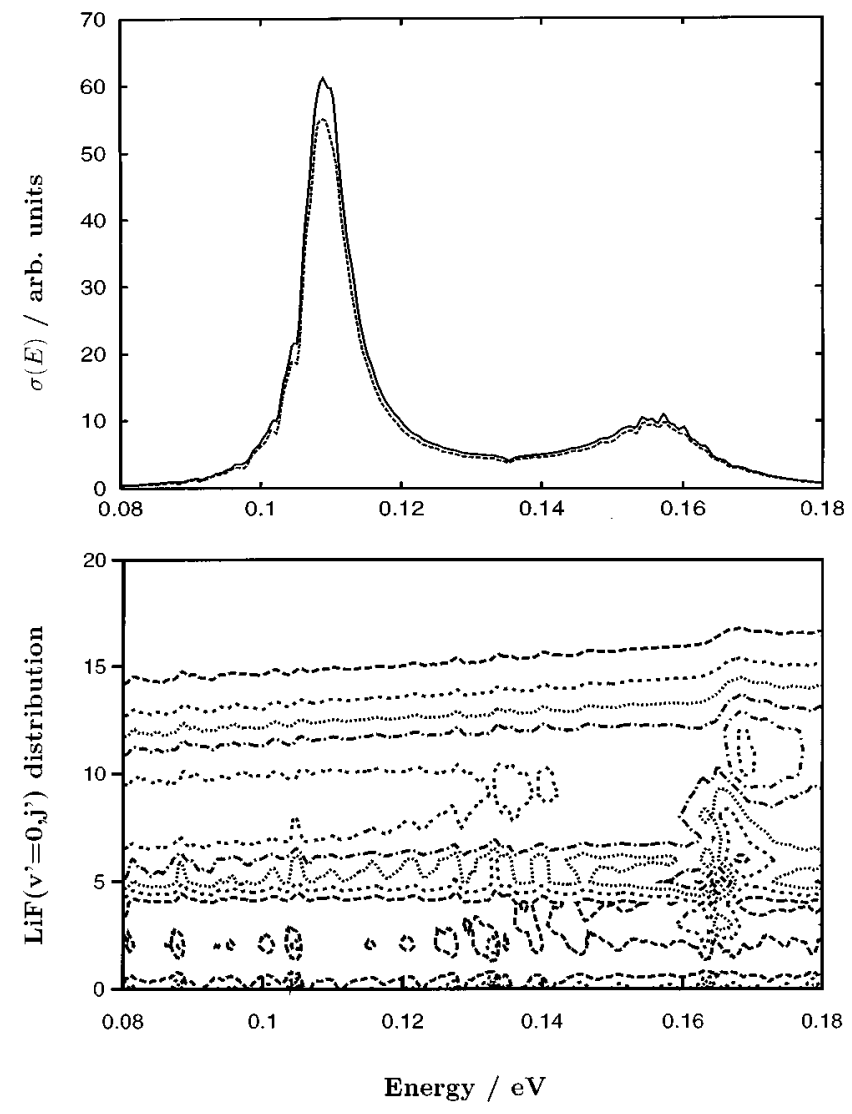

FIG. 3. $J_{i}=1 \rightarrow J=0$ transition. (a) Full line, absorption spectrum, $\sigma(E)$; dashed line, total reaction cross section, $\sigma^{\mathrm{LiF}}(E)$; and (b) final rotational distribution of $\operatorname{LiF}\left(v^{\prime}=0\right)$ obtained as $\sigma_{v=0, j}^{\mathrm{LiF}} / \Sigma_{j^{\prime}} \sigma_{v=0, j^{\prime}}^{\mathrm{LiF}}$ (the higher contour is of 0.12 and the increment is of -0.02 in the population normalized to 1 ).

also quite smooth, showing a slight increase of rotational excitation with energy, the most populated level being $j^{\prime}$ $\approx 10$ in all energy intervals. The weak energy dependence of the final $\operatorname{LiF}\left(v^{\prime}, j^{\prime}\right)$ distribution and the fast convergence with time of the partial cross section on the products, indicates that the dissociation in the products channel is essentially a direct process. The initial wave packet mainly corresponds to a $v=1$ excitation in the HF vibrational mode. The mass of $\mathrm{H}$ is rather small as compared to that of $\mathrm{F}$ and, hence, the vibration of $\mathrm{H}$ with respect to that of $\mathrm{LiF}$ in the initial wave packet is also in $v=1$. Therefore, the square of the overlap between the initial wave packet and the dissociative continua describing the $\mathrm{H}-\mathrm{LiF}$ fragmentation (which is approximately proportional to the absorption cross section ${ }^{23}$ ) shows two peaks as a function of energy associated to the two typical maxima associated to a $v=1$ state. The fact that the $\mathrm{LiF}$ products are rotationally excited is an evidence that the initial wave packet has an important excitation in the bending mode.

The situation is quite different for the $\operatorname{HF}(v=0, j)$ distribution which has a complicated structure with many narrow peaks associated to resonances, which also appeared in the reaction probability in the $\operatorname{Li}+\mathrm{HF}(v=0)$ full collision calculation recently reported using the same PES. ${ }^{19}$ The HF is also rotationally excited, but $\sigma_{v j}^{\mathrm{HF}}(E)=0$ for $j>7$ in the energy interval considered because of the high rotational constant of $\mathrm{HF}\left(\approx 21 \mathrm{~cm}^{-1}\right)$.

The scene changes in the course of a full collision, for which the reaction probability, in Ref. 19, is of the order of $10 \%-20 \%$ for different initial rotational states of $\operatorname{HF}(v$ $=0$ ) and $J=0$. However, at higher energies and for $v=1$ the collision is very efficient, ${ }^{24}$ the reaction probability at $J=0$ being of the order $80 \%-90 \%$. This factor of $\approx 4$ between the reaction probabilities obtained between $v=0$ and 1 increases to $10-50$ when comparing cross sections since the number of partial waves contributing for $v=1$ is larger as a consequence of the higher energy. This important enhancement of the reactivity is in excellent agreement with the experimental data available for related systems and is a consequence of a late barrier.

The high efficiency of the reaction in the photoinduced process is therefore explained in similar terms. The HF distance is slightly longer in the complex formed in the well of the reactants valley, $0.939 \AA$, than for free $\mathrm{HF}, 0.921 \AA$, while at the transition state it is $1.301 \AA$. Excitation of this mode promotes reaction with high efficiency. However, during the approach of the reactant in a collision it does not seem probable that the HF vibration gets excited, and it needs to be initially prepared in $v=1$ to achieve a high reaction efficiency.

The experimental detection of the products is however complicated because during the formation of the complex in a supersonic expansion some reactive collisions take place. However, such collisions are produced in the high density regime and, therefore, the $\mathrm{LiF}$ products are expected to be quite cold. This inconvenience disappears in some related systems for which the reaction in endothermic for initial $v$ $=0$ and the reactants need to be vibrationally excited to produce the reaction, as it is the case of Ca-HF. ${ }^{14,25}$

We want to thank Dr. Marta I. Hernández and Dr. José Campos-Martínez for very fruitful discussions. This work has been supported by DGICYT (Ministerio de Educación y Ciencia, Spain) under Grant Nos. PB94-0160 and PB950071. We also want to acknowledge DGICYT and CIEMAT for the use of a CRAY-J90.

${ }^{1}$ R. B. Metz, S. E. Bradforth, and D. M. Neumark, Adv. Chem. Phys. 81, 1 (1992).

${ }^{2}$ S. E. Bradforth, D. W. Arnold, D. M. Neumark, and D. E. Manolopoulos, J. Chem. Phys. 99, 6345 (1993).

${ }^{3}$ M. Dantus, R. M. Bowman, M. Gruebele, and A. H. Zewail, J. Chem. Phys. 91, 7437 (1989).

${ }^{4}$ N. F. Scherer, C. Sipes, R. B. Bernstein, and A. H. Zewail, J. Chem. Phys. 92, 5239 (1990).

${ }^{5}$ B. Soep, C. J. Whitham, A. Keller, and J. P. Visticot, Faraday Discuss. Chem. Soc. 91, 191 (1991).

${ }^{6}$ B. Soep, S. Abbés, A. Keller, and J. P. Visticot, J. Chem. Phys. 96, 440 (1992).

${ }^{7}$ S. K. Shin, Y. Chen, S. Nckolaisen, S. W. Sharpe, R. A. Beaudet, and C. Wittig, Adv. Photochem. 16, 249 (1991).

${ }^{8}$ K. Liu, J. C. Polanyi, and S. Yang, J. Chem. Phys. 98, 5431 (1993).

${ }^{9}$ J. C. Polanyi and J-X. Wang, J. Phys. Chem. 99, 13691 (1995).

${ }^{10}$ R. A. Loomis, R. L. Schwartz, and M. I. Lester, J. Chem. Phys. 104, 6984 (1996).

${ }^{11}$ R. D. Levine and R. B. Bernstein, Molecular Reaction Dynamics and Chemical Reactivity (Oxford University Press, Oxford, 1987).

${ }^{12}$ T. J. Odiorne, P. R. Brooks, and J. V. V. Kasper, J. Chem. Phys. 55, 1980 (1971). 
${ }^{13}$ J. G. Pruett and R. N. Zare, J. Chem. Phys. 64, 1774 (1976).

${ }^{14}$ Z. Karny and R. N. Zare, J. Chem. Phys. 68, 3360 (1978).

${ }^{15}$ M. Hoffmeister, R. Schleysing, F. Stienkemeier, and H. J. Loesch, J. Chem. Phys. 90, 3528 (1989).

${ }^{16}$ J. C. Polanyi and W. H. Wong, J. Chem. Phys. 51, 1439 (1969).

${ }^{17}$ M. H. Mok and J. C. Polanyi, J. Chem. Phys. 51, 1451 (1969).

${ }^{18}$ A. Aguado, M. Paniagua, M. Lara, and O. Roncero, J. Chem. Phys. 106, 1013 (1997)

${ }^{19}$ A. Aguado, M. Paniagua, M. Lara, and O. Roncero, J. Chem. Phys. 107, 10085 (1997).
${ }^{20}$ C. H. Becker, P. Casavecchia, P. W. Tiedemann, J. J. Valentini, and Y. T. Lee, J. Chem. Phys. 73, 2833 (1980).

${ }^{21}$ A. Aguado, C. Suárez, and M. Paniagua, Chem. Phys. 201, 107 (1995).

${ }^{22}$ G. G. Balint-Kurti, R. N. Dixon, and C. C. Marston, J. Chem. Soc., Faraday Trans. 86, 1741 (1990).

${ }^{23}$ R. Schinke, Photodissociation Dynamics, Spectroscopy and Fragmentation of Small Molecules (Cambridge University Press, Cambridge, 1993).

${ }^{24}$ M. Lara, O. Roncero, A. Aguado, and M. Paniagua (in preparation).

${ }^{25}$ R. L. Jaffe, M. D. Pattengill, F. G. Mascarello, and R. N. Zare, J. Chem. Phys. 86, 6150 (1987). 